\title{
Escala de funcionamiento familiar Propiedades psicométricas modificadas en una muestra mexicana
}

Modified family functioning scale: Psychometric properties in a Mexican sample

(c)
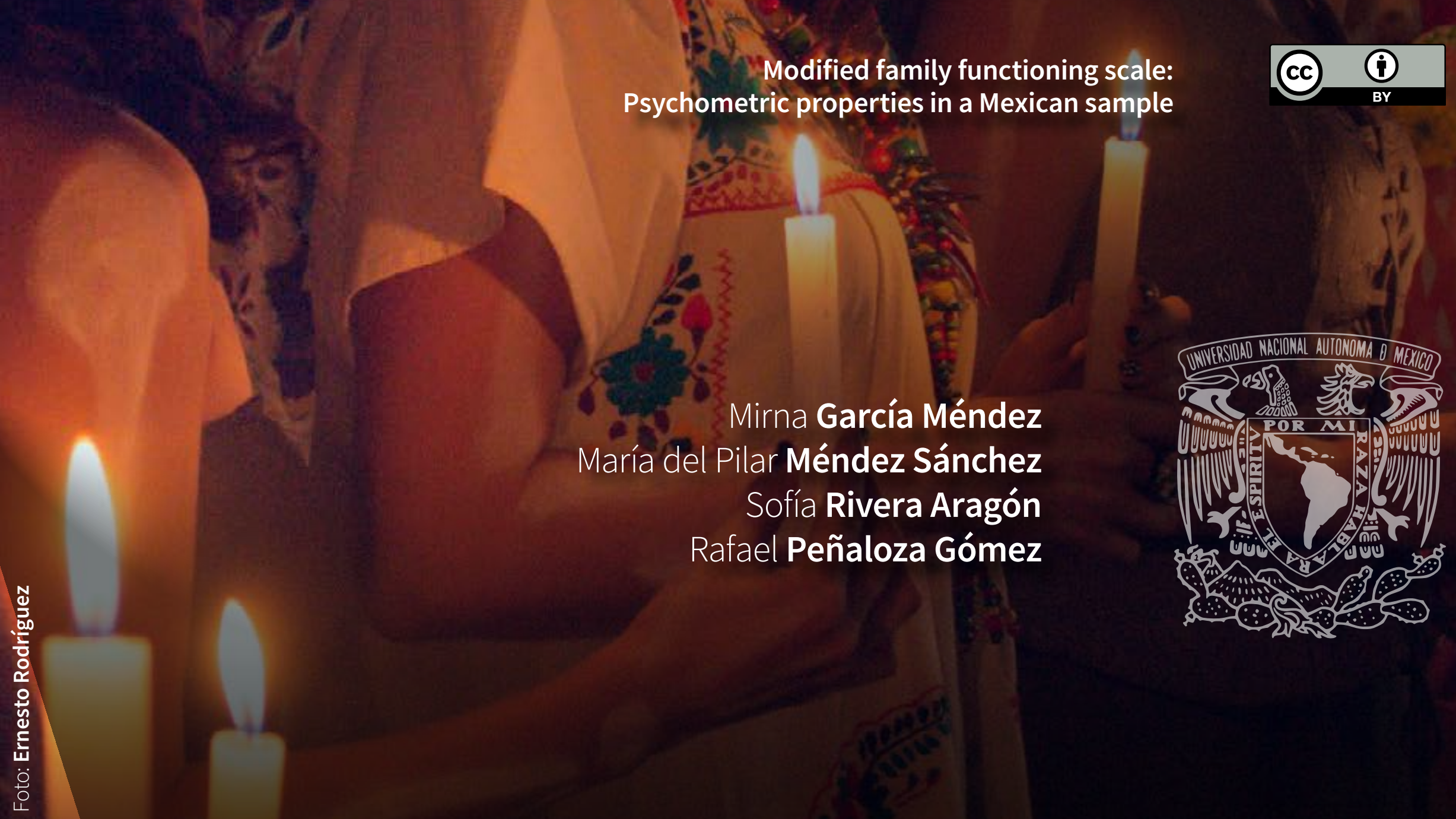

Revista Iberoamericana de

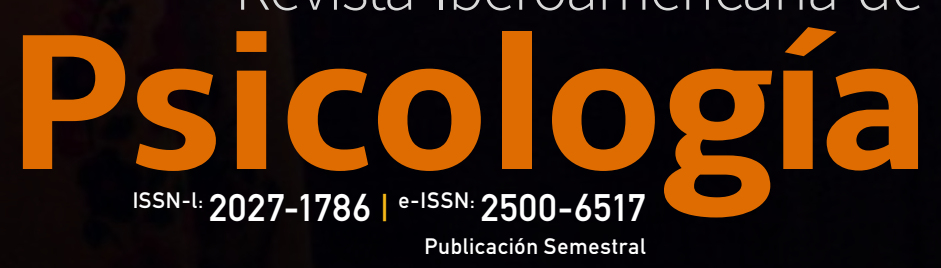


Revista Iberoamericana de

Psicología

ISSN-I: 2027-1786 | e-ISSN: 2500-6517

Publicación Semestral

ID:

2027-1786.rip.10103

Title: Modified family functioning scale

Subtitle: Psychometric properties in a Mexican sample

Título: Escala de funcionamiento familiar

Subtítulo: Propiedades psicométricas modificadas en una muestra mexicana

Alt Title / Título alternativo:

[en]: Psychometric properties of the modified family functioning scale, a Mexican sample

[es]: $\quad$ Propiedades Psicométricas de la Escala de Funcionamiento Familia Modificada en una Muestra Mexica

Author (s) / Autor (es):

García Méndez, Méndez Sánchez, Rivera Aragón, \& Peñaloza Gómez

Keywords / Palabras Clave:

[en]: family; factor analysis; support; problems; psychometry

[es]: familia; análisis factorial; apoyo; problemas; psicometría

Financiación / Funding:

Programa UNAM-DGAPA-PAPIIT clave IN306616

Submited: 2017-04-16

Acepted: 2017-07-17

\section{Resumen}

El objetivo de este estudio fue validar las propiedades psicométricas de la versión modificada de la Escala de Funcionamiento Familiar (García Méndez, 2006). Colaboraron de manera voluntaria 1196 participantes de la Ciudad de México: 471 padres (39.4\% de la muestra) y 725 madres (60.6\% de la muestra), con un rango de edad de 25 a 43 años ( $M=34$, DT = 5.10). La muestra fue no probabilística y se dividió en dos grupos con igual número de integrantes cada uno. Con un grupo de 598 participantes, se realizó un análisis factorial exploratorio que agrupó 32 ítems distribuidos en cinco factores, los cuales evalúan el funcionamiento familiar. La varianza total explicada fue de $44.08 \%$ y la consistencia interna global de la escala fue de 0.79. Los factores fueron: ambiente familiar positivo ( $\alpha=.885)$, conflicto ( $\alpha=$ .848), diversión ( $\alpha=.791$ ), hostilidad ( $\alpha=$ .721) y coaliciones $(\alpha=.660)$. Para probar la estructura de la escala se realizó un análisis factorial confirmatorio con esos 598 participantes. Los valores obtenidos en los ajustes NFI, CFI, NFI y RMSEA demostraron la estabilidad del modelo con los 5 factores. Se concluyó que la escala cumple con las

propiedades psicométricas requeridas para evaluar el funcionamiento familiar en población mexicana.

\section{Abstract}

The aim of this study was to validate the

psychometric properties of the modified

version of the Family Functioning Scale

(García Méndez, 2006). 1196 people from

Mexico City participated voluntarily in the study: 471 fathers (39.4\% of the sample) and

725 mothers ( $60.6 \%$ of the sample), with ages between 25 and 43 years old ( $M=34$, $\mathrm{SD}=5.10)$. The sample was non-probabilistic and it was divided into two groups (with the same number of participants per group). An exploratory factor analysis was performed

with one group of 598 participants. This analysis grouped 32 items distributed within five factors that evaluate family functioning.

The total explained variance was $44.08 \%$ and the overall internal consistency of the scale was 0.79 . The factors were: positive family environment ( $\alpha=0.885)$, conflict ( $\alpha$ $=0.848)$, fun ( $a=0.791)$, hostility ( $a=0.721$ ) and coalitions $(a=0.660)$. A confirmatory factor analysis was carried out with those 598 participants in order to test the scale structure. The values obtained in the NFI, CFI, NFI and RMSEA adjustments demonstrated the stability of the model with the 5 factors.

It was concluded that the scale fulfills the psychometric properties required to evaluate family functioning in the Mexican population.

\author{
Lic Mirna García Méndez, [Dr] \\ ORCID: $\quad$ 0000-0002-2334-0740 \\ Source I Filiacion: \\ Universidad Nacional Autónoma de México \\ BIO: \\ Facultad de Estudios Superiores Zaragoza \\ UNAM, Universidad Nacional Autónoma de \\ México \\ City I Ciudad: \\ México DF $[\mathrm{mx}]$ \\ e-mail: \\ mina@unam.mx
}

\section{Lic Sofía Rivera Aragón, [Dr] \\ ORCID: $\quad$ 0000-0003-1170-2430}

Source I Filiacion:

Universidad Nacional Autónoma de México BIO:

Facultad de Estudios Superiores Zaragoza UNAM, Universidad Nacional Autónoma de México

City I Ciudad:

México DF $[\mathrm{mx}]$

e-mail:

sofiar@unam.mx

\author{
Lic María del Pilar Méndez Sánchez, [Dr] \\ ORCID: 0000-0003-0066-5582 \\ Source I Filiacion: \\ Universidad Nacional Autónoma de México \\ BIO: \\ Facultad de Estudios Superiores Zaragoza \\ UNAM, Universidad Nacional Autónoma de \\ México \\ City I Ciudad: \\ México DF $[\mathrm{mx}]$ \\ e-mail: \\ mendezsanchezp@gmail.com
}

Lic Rafael Peñaloza Gómez, [Dr]

ORCID: $\quad$ 0000-0003-3585-7701

Source I Filiacion:

Universidad Nacional Autónoma de México BIO:

Facultad de Estudios Superiores Zaragoza UNAM, Universidad Nacional Autónoma de México

City I Ciudad:

México DF $[\mathrm{mx}]$

e-mail:

penaloza.gr@gmail.com

Citar como:

García Méndez, M., Méndez Sánchez, M. d., Rivera Aragón, S., \& Peñaloza Gómez, R. (2017). Escala de funcionamiento familiar: Propiedades psicométricas modificadas en una muestra mexicana. Revisto Iberoamericana de Psicología issn-l:2027-1786, 10 (1),19-27.

Obtenido de: https://revistas.iberoamericana.edu.co/index.php/ripsicologia/article/view/1187 


\section{Escala de funcionamiento familiar en una muestra mexicana}

Propiedades psicométricas modificadas

Modified family functioning scale: psychometric properties in a Mexican sample

Mirna García Méndez
María del Pilar Méndez Sánchez
Sofía Rivera Aragón
Rafael Peñaloza Gómez

\section{Introducción}

La familia es el grupo primario básico que pertenece una persona, en el que se mantienen relaciones recíprocas, pautadas y redundantes. En tanto unidad social, mantiene las raíces sociales y culturales, a la vez que enfrenta múltiples tareas de desarrollo, desempeñadas a partir de los parámetros propios de cada cultura (Minuchin, 1974/1995). Los integrantes de la familia, día a día, participan en dichas tareas de manera activa y sus interacciones se organizan de manera dinámica en patrones de interacción semi-regulares, de modo tal que a través de estas interacciones son afectados y a su vez afectan a otros (Larson \& Almeida, 1999), es decir, se afectan los miembros que conforman la familia así como otras personas externas a ésta, lo anterior, debido a un nexo de intercambio diario entre sus miembros, y entre éstos y sus ambientes externos, por ejemplo el lugar de trabajo de los padres, la escuela de los hijos y otras instituciones de la comunidad. (Larson \& Almeida, 1999)

De acuerdo con lo anterior, la familia es entonces un sistema dinámico vivo que se encuentra en transformación constante a través del tiempo con el propósito de asegurar la continuidad y el crecimiento psicosocial de sus miembros (Andolfi, Angelo, Menghi, \& Nicolò Corigliano, 2007; McGoldrick \& Gerson, 1993/2009). Esta transformación constante se encuentra matizada por el contexto socio-histórico del momento, en tanto la familia funciona en contextos sociales específicos. Así pues, la familia constituye un sistema sociocultural en transformación, cuyos cambios implican un desplazamiento a través de etapas que exigen una reestructuración, lo cual la dota de cierta flexibilidad y adaptabilidad en tanto su capacidad de ajustarse a las circunstancias cambiantes, siempre manteniendo o procurando su continuidad, cohesión y promoviendo el crecimiento psicosocial de sus integrantes (Minuchin, 1974/1995).

\section{Dinámicas Familiares}

Estas dinámicas que se dan al interior de la familia determinan el establecimiento de pautas de interacción entre sus miembros, condición que imprime un significado particular a su estructura y dinámica. Así pues, los cambios experienciados por cualquiera de sus miembros, díada o tríada ${ }^{1}$, desencadenan en períodos de desequilibrio, durante los cuales las familias co-regulan sus relaciones con el fin de llegar a un nuevo estado de equilibrio (Everri, Fruggeri, \& Molinari, 2014), lo que modifica el ciclo de vida de las personas que la integran (Weston \& Qu, 2014). De lo anterior se sigue que las experiencias vividas en la familia establecen un legado que influye en sus valores y orientaciones, determinando las pautas de comportamiento con los demás y funcionando como base o modelo para muchas de las elecciones que toman sus integrantes respecto a sus propias vidas. (Anderson \& Sabatelli, 2003)

En cuanto a las normas que rigen a la familia, como la afiliación y la abnegación, éstas se relacionan con el familismo y el respeto a los padres, pautas de convivencia que distinguen a la familia mexicana como parte de una cultura colectivista (Díaz Guerrero, 1994). Existen pues un conjunto de premisas histórico-socio-culturales (PHSC) donde la figura de la madre es enaltecida, en tanto encargada de proporcionar amor a los miembros de la familia. En consonancia con lo anterior, se le asocia con la abnegación y el autosacrificio (Díaz Guerrero, 2003), pues este amor incondicional supone anteponer los intereses de la familia sobre los intereses personales. Por otro lado, la figura del padre se asocia con la supremacía y el poder, obteniéndose así una mezcla de amor y poder al interior de la familia (Díaz Guerrero, 1994; 2003). Estas normas y premisas se han retomado en el tratamiento terapéutico con familias

En tanto en el curso de la vida familiar intervienen elementos asociados con lazos sociales estrechos: la díada padre-hijo, la relación entre hermanos y la díada conyugal, entre otros. (David Barrett, y otros, 2016) 


\section{Escala de funcionamiento familiar}

Propiedades psicométricas modificadas en una muestra mexicana

latinas, haciendo énfasis en el marianismo, el respeto y el familismo, asumido como la cercanía familiar, la colaboración y la unidad. (Zafra, 2016)

En consonancia con lo anterior, al interior de la familia se establecen lazos que mantienen la identidad y privacidad, se llevan a cabo acciones con el propósito de lograr una estabilidad relacionada con el compromiso, la seguridad y la socialización de sus miembros (Donalek, 2009). Así, cuando se presenta algún problema, la persona implicada se describe a sí misma como 'favorecida por su familia' (Pernice Duca, 2010), la cual, como institución, tiene responsabilidad sobre el bienestar de sus miembros, y de manera indirecta de la comunidad, proveyendo a sus integrantes las bases para una buena salud y para el ejercicio de prácticas saludables de bienestar (Ackerman, 1995; Garst, Baughman, Franz, \& Seidel, 2013; Lo Presti, D’Aloisio, \& Pluviano, 2016; Williams, 2003). Por ejemplo, el desarrollo de relaciones seguras entre padres e hijos impacta las interacciones con grupos externos y el desarrollo de habilidades (Matheson, y otros, 2005). De acuerdo con lo anterior, en las relaciones familiares el ambiente familiar positivo es un elemento esencial, en el que la comunicación y la cohesión favorecen la cercanía, la expresión de sentimientos positivos, la empatía y la salud familiar. (García Méndez, Rivera Aragón, Reyes Lagunes, \& Díaz Loving, 2006; Olson, 1991; Olson, 2000; Olson, Russell, \& Sprenkle, 1983; Schrodt, 2005)

Sin embargo, existen a su vez patrones de interacción que provocan tensiones al interior de la familia, como la evitación (que se relaciona con la dificultad para resolver problemas, con la falta de involucramiento y de atención). Aunado a las dificultades para resolver problemas, otra variable que afecta el funcionamiento familiar es la naturaleza de las experiencias de los cónyuges y los triángulos primarios en sus familias de origen (las tres partes del triángulo principal frecuentemente las integran: el niño, la madre y el padre, o el niño, los padres y otro miembro de la familia (Klever, 2009).

Dependiendo de la intensidad, frecuencia y contenido, el conflicto marital es otro factor que afecta de manera negativa a los integrantes de la familia, principalmente a los hijos (Fainsilber Katz \& Woodin, 2002). Estos elementos favorecen la presencia de patrones de interacción disfuncionales que pueden provocar límites rígidos que afectan la tendencia al cambio y adaptación, a tal punto que los integrantes de la familia pueden presentar serias dificultades para cambiar a medida que las etapas del ciclo de vida así lo requieran. Estos eventos se asocian con familias demasiado rígidas que no propician ni permiten

Tabla 1 Instrumentos que evalúan el funcionamiento familiar

Instrumento
Cuestionario de funcionamiento familiar
(Atri \& Zetune, 1987)

Conformado por $\mathbf{4 0}$ ítems y $\mathbf{6}$ factores: involucramiento afectivo funcional, involucramiento afectivo disfuncional, patrones de comunicación disfuncionales, patrones de comunicación funcionales, resolución de problemas, patrones de control de conducta. $\alpha=0.92$, varianza no reportada

ningún cambio, o con familias demasiado fragmentadas que están en peligro de dispersión (Hoffman, 1992), que favorecen interacciones distantes acompañadas por falta de demostraciones de afecto.

\section{Funcionamiento familiar}

Retomando los planteamientos anteriores, las relaciones entre los miembros de la familia se convierten en pautas de interacción vinculadas al funcionamiento familiar, las cuales se caracterizan tanto por la naturaleza de las relaciones mismas, como por a la influencia de los ambientes externos en los que la familia se desenvuelve. Dichas relaciones familiares implican movimiento y cambio, y el funcionamiento familiar está estrechamente vinculado a estos dos aspectos que, a su vez, implican la capacidad de sus integrantes para llevar a cabo modificaciones y ajustes a sus modos de interacción, de tal manera que respondan a sus propias necesidades y a la etapa del ciclo de vida que estos atraviesen.

Algunos de los elementos asociados al cambio tienen que ver con la estructura de la familia, sus límites y reglas, demostraciones afectivas y cogniciones, con las historias de vida de sus integrantes, y con la cultura a la que pertenecen (Anderson \& Sabatelli, 2003; Díaz Loving, 2004). En el caso de México, el sentido de pertenencia y de afiliación a la familia es un factor importante en su funcionamiento, el cual se refleja en la convivencia cotidiana, la recreación, la comunicación, la cohesión y el apoyo entre sus miembros. Estos aspectos se ven fortalecidos gracias a eventos (bodas, bautizos, funerales, entre otros) que se transmiten a través de la cultura y constituyen símbolos universales que ofrecen la oportunidad de aclarar y afianzar el rol o posición que se tiene al interior de la familia y de hacer valer su identidad colectiva. (Bennett, 1991)

De acuerdo con lo anterior, las relaciones familiares pueden ser favorables o desfavorables en tanto las experiencias en la familia son unas de las fuentes más importantes de bienestar y malestar, en tanto son particularmente importantes para el desarrollo de las habilidades interpersonales y de los lazos sociales (Vandeleur, Jeanpretre, Perrez, \&Schoebi, 2009). Estas interacciones entre los miembros de la familia, asociadas al cambio, toman lugar en dos dimensiones, a saber: coordinación y oscilación, la primera se asocia a la continuidad familiar y mantiene vivas las relaciones, la segunda refiere al cambio, se expresan viejas y nuevas maneras de interactuar y comunicarse. (Everri, Fruggeri, \& Molinari, 2014)
Escala de evaluación de recursos familiares (FAD) (Epstein, Baldwin, \& Bishop, 1983)

Escala de evaluación de la adaptabilidad y cohesión familiar (FACES IV)

(Olson, FACES IV and the circumplex model: Validation study., 2011)

Escala de funcionamiento familiar (Palomar, 1999).

Escala de evaluación de las Relaciones Intrafamiliares (E.R.I.) (Rivera Heredia \& Andrade Palos, 2010)

Escala de ambiente familiar (FES) (Moos, Insel, \& Humprey, 1974)
60 reactivos distribuidos en siete sub-escalas Tiene un $\alpha=0.70$ a 0.90 y una confiabilidad test-re-test de $\mathbf{0 . 6 6}$ a $\mathbf{0 . 7 6}$

\section{Contiene $\mathbf{4 2}$ ítems:}

$\mathbf{2 1}$ para la escala de cohesión (tres factores: enmarañada, separada y balanceada) 21 para la escala de flexibilidad (tres factores: caótica, rígida y balanceada) Los valores alpha de Cronbach oscilan entre 0.77 y 0.89

$\mathbf{4 6}$ reactivos y 10 factores. La varianza explicada es de $\mathbf{5 3} \%$. Sus índices de confiabilidad van de $\mathbf{0 . 5 2}$ a $\mathbf{0 . 9 4}$

Conformada por 56 ítems y 3 factores (unión y apoyo, expresión y dificultades) que explican el $\mathbf{5 7 \%}$ de la varianza, La confiabilidad va de $\mathbf{0 . 9 2}$ a $\mathbf{0 . 9 5}$

Conformada por $\mathbf{9 0}$ ítems de falso-verdadero, distribuidos en $\mathbf{1 0}$ subescalas que conforman tres dimensiones: de relación (subescalas cohesión, expresividad, conflicto), de crecimiento personal (subescalas independencia, orientación al logro, orientación intelectual-cultural, orientación recreativa-activa, énfasis moral-religioso) y de mantenimiento del sistema (subescalas organización y control). La confiabilidad varía de $\mathbf{0 . 6 1}$ a $\mathbf{0 . 7 8}$
Dimensiones que evalúa

Evalúa la percepción de un miembro de la familia sobre el funcionamiento familiar, con la finalidad de identificar áreas de conflicto.

Dimensiones del modelo McMaster.

Evalúa la cohesión y flexibilidad familiar

Percepción global del funcionamiento familiar

Evalúa las relaciones intrafamiliares en jóvenes

Evalúa el ambiente familiar 
En el funcionamiento familiar influyen varios aspectos y elemento, por ejemplo, las habilidades positivas de comunicación (Martin \& Martin, 2000), asociadas con la cercanía, la atención y el apoyo, que le permiten a la familia compartir sus necesidades y preferencias. Por otro lado, en contraste, mensajes ambiguos y la crítica contribuyen al establecimiento de patrones de interacción negativos, que reducen la capacidad de sus miembros para compartir sus sentimientos (Olson, 2000). En este proceso, el apoyo de la familia, visible en la expresión de aceptación, apreciación, reconocimiento, elogios y aliento, está negativamente relacionado a la crítica de otros miembros de la familia (Russel, 1979).

Otros factores importantes que influye en el funcionamiento familiar son las reglas y límites establecidos y que modulan las interacciones, los cuales se vuelven rutinarios y se convierten en principios que gobiernan la vida familiar (Anderson \& Sabatelli, 2003). Dichos límites deben ser claros y precisos para que cada quien pueda llevar a cabo sus funciones sin interferir con las de los otros y siempre manteniendo la cercanía con los demás integrantes de la familia (Minuchin, 1974/1995). Estos elementos se asumen como patrones recurrentes de interacción que definen los límites de las conductas aceptables y apropiadas en la familia, reflejan sus valores como sistema y definen los roles de sus miembros (Anderson \& Sabatelli, 2003).

Debido a las diversas variables implicadas y a la intrínseca complejidad de la familia como sistema, la evaluación de su funcionamiento resulta complejo (Larraín, Zegers, Díez, Trapp, \& Polaino Lorente, 2003), lo que deriva en una la amplia elaboración de diversos instrumentos de medición, algunos de los cuales se presentan en la Tabla 1.

Queda claro, a partir de las secciones anteriores, que en el funcionamiento familiar intervienen múltiples factores, por ello es considerado un constructo multifactorial y, en consecuencia, existen diferentes instrumentos que evalúan distintos aspectos de este constructo. La Tabla 1 muestra las propiedades de algunos de esos instrumentos. Para el caso específico de la cultura mexicana, se consideraron tres instrumentos:

\section{Atri y Zetune (1987) cuya muestra fue de nivel socioeconómico alto \\ Rivera Heredia y Andrade Palos (2010) para jóvenes \\ Palomar (1999) que evalúa el funcionamiento familiar general.}

Cada uno de los instrumentos citados tiene características específicas, por lo que su elección depende de los objetivos y alcances de la investigación.

En el García Méndez, Rivera Aragón, Reyes Lagunes y Díaz Loving (2006), elaboraron una escala Likert que evalúa indicadores positivos y negativos de las interacciones familiares. Dicha escala contiene $\mathbf{4 5}$ ítems distribuidos en cuatro factores:
ambiente familiar positivo
hostilidad/evitación del conflicto
mando/problemas en la expresión de sentimientos
cohesión/reglas.

El análisis factorial empleado en esta versión fue el de componentes principales, con el que se obtuvo una varianza explicada de $\mathbf{5 6} \%$ y un índice de fiabilidad general de $\mathbf{0 . 8 5}$. Algunas de las investigaciones en las que se ha empleado la escala tienen que ver con: la identificación de la relación entre el funcionamiento familiar con la resiliencia materna (Roque Hernández \& Acle Tomasini, 2013), los problemas de consumo de alcohol (García Cortés, García Méndez, \& Rivera Aragón, 2015), la migración y la cultura (Toledano Toledano, 2010), la discapacidad de los hijos (Rea Amaya, Acle Tomasini, Ampudia Rueda, \& García Mendez, 2014), y la predicción de la alexitimia (Rojas Ramírez \& García Méndez, 2016).
Con el propósito de evaluar de una manera más integral el funcionamiento familiar, se elaboró una versión modificada de la escala, la cual incorpora nuevos ítems a la vez que suprimió los que tenían doble negación, en tanto generaban confusión en los participantes al momento de ser respondidos. En consonancia con lo anterior, el objetivo de este estudio fue analizar las propiedades psicométricas de la Escala de Funcionamiento Familiar Modificada (EFFAMO), con la finalidad de contar con un instrumento válido y confiable para la evaluación de las relaciones familiares, que pueda ser utilizado en el área clínica y en la investigación en torno a la familia.

\section{Método}

\section{Participantes}

Colaboraron $\mathbf{1 1 9 6}$ participantes voluntarios: $\mathbf{4 7 1}$ padres (correspondientes al $\mathbf{3 9 . 4 \%}$ de la muestra) y $\mathbf{7 2 5}$ madres (correspondientes al $\mathbf{6 0 . 6 \%}$ de la muestra) de la Ciudad de México, cuyo rango de edad fue de 25 a 43 años $(\mathbf{M}=34$, DT = 5.10). De éstos $\mathbf{1 1 9 6}$ participantes, $\mathbf{8 2 2}$ estaban casados (68.7\%) y $\mathbf{3 7 4}$ co-habitaban (31.2\%). El número de hijos que tenían era entre uno y cinco (Mo =2). En lo que respecta a sus ocupaciones, $\mathbf{4 4 2}$ eran 'amas de casa' (36.9\%), 360 eran 'empleados' (30.1\%), 114 'con oficio' (9.5\%), 121 'comerciantes' (10.1\%), 105 'profesionistas' (8.8\%), $\mathbf{1 3}$ 'estudiantes' (1.1\%), 27 estaban 'desempleados' (2.3\%), y $\mathbf{1 4}$ 'técnicos' (1.2\%). El tiempo que los participantes habían dedicado a sus estudios oscilaba entre 1 y 23 años $(M=6.09, D T=4.53)$, lo que en términos del nivel de escolaridad equivaldría a 'educación básica'. Por último, es preciso agregar que la muestra fue no-probabilística intencional.

\section{Instrumento}

El instrumento empleado fue le Escala de Funcionamiento Familiar Modificada (EFFAMO). Se utilizó una versión modificada, basada en la original de García-Méndez et al. (2006) (descrita anteriormente). La EFFAMO está compuesta por $\mathbf{4 0}$ ítems y $\mathbf{5}$ intervalos de respuesta (desde 1 = "totalmente en desacuerdo" a 5 = "totalmente de acuerdo").

\section{Procedimiento}

Dado que los participantes se localizaban en diferentes puntos de la Ciudad de México D.F. (sus hogares, escuelas y otros lugares públicos), fue preciso hablar previamente con las autoridades respectivas con el fin de contar con su aprobación para llevar a cabo la aplicación del instrumento en las escuelas, de ser posible. El objetivo de la investigación se socializó y se explicó a aquellos quienes accedieron a colaborar de manera voluntaria y sin remuneración alguna a cambio de su participación. Posteriormente se llevó a cabo la firma del consentimiento informado, se hizo entrega de la escala en cuestión y se les informó que no habría tiempo límite para responderla. En este punto, se hizo especial énfasis en la importancia del anonimato y la confidencialidad de la información, así como en el hecho que no había respuestas 'buenas/correctas' o 'malas/incorrectas'.

La aplicación en las escuelas se llevó a cabo de manera grupal, en los espacios y horarios asignados por los directivos de las mismas. En los hogares y lugares públicos la aplicación se realizó de manera individual y, en ambos casos, los investigadores y becarios colaboraron 


\section{Escala de funcionamiento familiar}

Propiedades psicométricas modificadas en una muestra mexicana

respondiendo todas las dudas de los participantes. La investigación se llevóa cabo con la aprobación de la Comisión de Bioética yBioseguridad, de la Universidad Nacional Autónoma de México, Facultad de Estudios Superiores Zaragoza.

Tabla 2 Ponderación de factores con rotación varimax de la escala funcionamiento familiar nuclear

\begin{tabular}{|c|c|c|c|}
\hline Fact & $\#$ & Ítems & CF \\
\hline \multirow{11}{*}{ 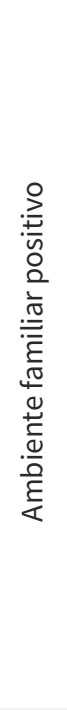 } & 15 & En mi familia, respetamos los intereses y gustos de todos & .759 \\
\hline & 14 & $\begin{array}{l}\text { Cuando hay un problema, los miembros de mi familia nos } \\
\text { reunimos y platicamos sobre las posibles soluciones }\end{array}$ & .674 \\
\hline & 6 & expresar nuestro afecto es algo importante para mi familia & .671 \\
\hline & 34 & $\begin{array}{l}\text { A los miembros de mi familia nos agrada consentirnos unos a } \\
\text { otros }\end{array}$ & .658 \\
\hline & 23 & $\begin{array}{l}\text { Los miembros de mi familia tenemos la libertad de decir lo que } \\
\text { nos agrada y nos molesta de los demás }\end{array}$ & .630 \\
\hline & 8 & A mi familia, nos agrada realizar cosas juntos & .623 \\
\hline & 16 & En la toma de decisiones familiares, participamos padres e hijos & .608 \\
\hline & 40 & En mi familia nos gusta pasar tiempo juntos & .529 \\
\hline & 21 & Mi familia se distingue por sus relaciones armoniosas & .505 \\
\hline & 3 & $\begin{array}{l}\text { En mi familia se buscan actividades recreativas en las que todos } \\
\text { podamos participar }\end{array}$ & .486 \\
\hline & 30 & En mi familia se platican cosas entre hermanos & .481 \\
\hline \multirow{9}{*}{ 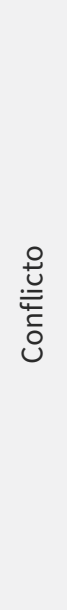 } & 4 & En mi familia los límites y reglas son poco claros & .650 \\
\hline & 37 & En mi familia se dice una cosa y se hace otra & .643 \\
\hline & 11 & $\begin{array}{l}\text { Los miembros de mi familia manifestamos, entre nosotros, } \\
\text { sentimientos diferentes a los que verdaderamente sentimos }\end{array}$ & .617 \\
\hline & 17 & En mi familia cada quien resuelve sus problemas como puede & .614 \\
\hline & 24 & $\begin{array}{l}\text { Las reuniones de mi familia ponen en evidencia las adicciones de } \\
\text { algunos de sus miembros }\end{array}$ & .604 \\
\hline & 10 & En mi familia se desconoce lo que cada uno espera del otro & .585 \\
\hline & 26 & $\begin{array}{l}\text { Mi familia comunica mensajes ambiguos, se pide una cosa } \\
\text { cuando en realidad se quiere que se haga otra }\end{array}$ & .551 \\
\hline & 5 & $\begin{array}{l}\text { Los integrantes de mi familia mostramos desinterés por los } \\
\text { demás }\end{array}$ & .538 \\
\hline & 2 & En mi familia nadie sigue la disciplina establecida por los papás & .522 \\
\hline \multirow{5}{*}{ 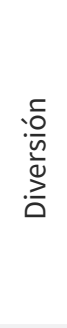 } & 20 & $\begin{array}{l}\text { En mi familia cualquier evento es bueno para celebrar e invitar a } \\
\text { nuestros amigos }\end{array}$ & .748 \\
\hline & 22 & En mi familia nos agrada reunirnos con nuestros amigos & .680 \\
\hline & 13 & En mi familia nos gusta organizar convivios y fiestas familiares & .605 \\
\hline & 18 & Mi familia tiene muchos amigos & .559 \\
\hline & 28 & $\begin{array}{l}\text { Mi familia asiste a reuniones sociales (fiestas, bodas, bautizos, } \\
\text { cumpleaños) }\end{array}$ & .472 \\
\hline \multirow{4}{*}{ 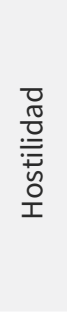 } & 35 & $\begin{array}{l}\text { En mi familia acostumbramos criticar a la persona que se } \\
\text { encuentra ausente }\end{array}$ & .534 \\
\hline & 33 & $\begin{array}{l}\text { En mi casa los miembros de la familia ocultamos lo que nos } \\
\text { desagrada de los demás }\end{array}$ & .509 \\
\hline & 7 & Los miembros de mi familia nos criticamos unos a otros & .482 \\
\hline & 32 & $\begin{array}{l}\text { A los miembros de mi familia nos desagrada convivir con } \\
\text { personas ajenas a nuestra familia }\end{array}$ & .402 \\
\hline \multirow{3}{*}{ 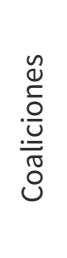 } & 12 & $\begin{array}{l}\text { En mi familia los hijos se ponen de acuerdo entre ellos, para } \\
\text { obtener alguna ganancia de mi pareja y de mí }\end{array}$ & .662 \\
\hline & 25 & $\begin{array}{l}\text { Mis hijos y mi pareja se unen para obtener algún beneficio de mi } \\
\text { persona }\end{array}$ & .602 \\
\hline & 39 & $\begin{array}{l}\text { Mis hijos y yo nos ponemos de acuerdo para obtener algún } \\
\text { beneficio de mi pareja }\end{array}$ & .516 \\
\hline
\end{tabular}

\section{Resultados}

Con el fin de conocer la distribución de las respuestas (normal o sesgada) se obtuvo la asimetría para cada ítem, la cual osciló entre

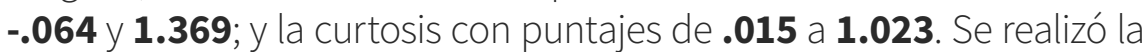
prueba $t$ de Student para grupos extremos, a partir de los percentiles $\mathbf{2 5}$ y $\mathbf{7 5}$, con la finalidad de conocer la discriminación de los ítems con una significancia menor a $\mathbf{. 0 0 1}$. Los resultados sugieren valores adecuados, por lo que se mantuvieron todos los ítems y se precedió a realizar una prueba de la medida de adecuación muestral de Kaiser-MeyerOlkin (KMO) (un índice para comparar la magnitud de los coeficientes de correlación observados con la magnitud de los coeficientes de correlación parcial). Considerando que para que el modelo factorial sea adecuado la correlación parcial entre las dos variables debe ser un valor próximo a 1, es posible afirmar que el KMO es adecuado, en tanto el valor obtenido fue de (0.901), además la Prueba de Esfericidad de Bartlett fue significativa $\left(X^{2}=6021.38, p<0.000\right)$.

Para conocer la estructura de la nueva escala, se llevó a cabo un análisis factorial de ejes principales con rotación ortogonal, mediante el método Varimax, minimizando el número de variables que tienen saturaciones altas en cada factor. En cada uno de los factores se consideraron las cargas factoriales iguales o mayores a $\mathbf{0 . 4 0}$, así como que los ítems puntuaran sólo en un factor. Resultado de este análisis, se eliminaron ocho ítems (1, 9, 19, 27, 29, 31, 36, 38), quedaron 32 distribuidos en cinco factores que explican una varianza total de $\mathbf{4 4 . 0 8 \%}$ (Tabla 2). Esta disminución de la varianza, en comparación con la primera versión de la escala, puede deberse a la sensibilidad que tiene el método de ejes principales al tamaño de la muestra. (de la Fuente Fernández, 2011)

El factor Ambiente Familiar Positivo se asume como la satisfacción con la relación y el intercambio de ideas que favorecen las interacciones familiares y las manifestaciones de afecto entre los miembros de la familia. El factor dos, Conflicto, se caracteriza por patrones de relación en los que prevalece el antagonismo y la no-solución de problemas. El factor tres, Diversión, se refiere a las actividades realizadas por los integrantes de la familia, asociadas con el esparcimiento. El factor cuatro, Hostilidad, se distingue por el predominio de malestar, desacuerdo y crítica hacia los demás, lo que limita la expresión de afecto entre los integrantes de la familia. El factor cinco, Coaliciones, se refiere a las alianzas entre los integrantes de la familia para obtener beneficios de otro(s). Las medias, desviaciones típicas y la varianza explicada por cada factor se presentan en la Tabla 3.

Tabla 3 Estadísticos descriptivos y la varianza explicada por cada factor de la escala de funcionamiento familiar

\begin{tabular}{|rccc|}
\hline Factores & $\begin{array}{c}\text { \% varianza } \\
\text { explicada }\end{array}$ & Media & $\begin{array}{c}\text { Desviación } \\
\text { Típica }\end{array}$ \\
\hline Ambiente familiar positivo & 22.51 & 3.77 & 0.79 \\
Conflicto & 11.71 & 1.95 & 0.75 \\
Diversión & 4.17 & 3.14 & 0.88 \\
Hostilidad & 2.94 & 2.04 & 0.83 \\
Coaliciones & 2.75 & 2.03 & 0.97 \\
\hline
\end{tabular}

La consistencia interna global de la EFAMO fue de $\mathbf{0 . 7 9}$. (Tabla 3). En cuanto a la correlación de cada ítem con el total de la escala, es importante señalar que sus puntuaciones superan el $\mathbf{0 . 4 5}$, de tal modo que se demuestra su aporte a la medición del constructo funcionamiento familiar. (Tabla 4)

Para confirmar la estructura de la EFFAMO se realizó un análisis factorial confirmatorio con el método de máxima verosimilitud, debido a que todas las variables del modelo cumplieron con el criterio de normalidad (de la Fuente Fernández, 2011). Los índices de ajuste obtenidos fueron: $X^{2}=1037.229, p<0.001 ; C F I=0.901, \| F I=0.903, N F I=.850$ y $R M S E A=.049$ ( $L O=.046$ y HI = .053). Estos valores indican ajustes adecuados del modelo (Hu \& Bentler, 1999) (Gráfico 1). 
Tabla 4 Consistencia interna de la escala de funcionamiento familiar

\begin{tabular}{|c|c|c|c|}
\hline$\#$ & Factor & CETC & $\alpha:[e]$ \\
\hline \multicolumn{4}{|c|}{ Ambiente familiar positivo (11 ítems) $\alpha=.885$} \\
\hline 15 & En mi familia respetamos los intereses y gustos de todos & .717 & .868 \\
\hline 14 & $\begin{array}{l}\text { Cuando hay un problema los miembros de mi familia nos } \\
\text { reunimos y platicamos sobre las posibles soluciones }\end{array}$ & .676 & .870 \\
\hline 6 & El expresar nuestro afecto es algo importante para mi familia & .624 & .874 \\
\hline 34 & $\begin{array}{l}\text { A los miembros de mi familia nos agrada consentirnos unos a } \\
\text { otros }\end{array}$ & .642 & .873 \\
\hline 23 & $\begin{array}{l}\text { Los miembros de mi familia tenemos la libertad de decir lo } \\
\text { que nos agrada y nos molesta de los demás }\end{array}$ & .623 & .874 \\
\hline 8 & A mi familia nos agrada realizar cosas juntos & .650 & .873 \\
\hline 16 & $\begin{array}{l}\text { En la toma de decisiones familiares, participamos padres e } \\
\text { hijos }\end{array}$ & .575 & .878 \\
\hline 40 & En mi familia nos gusta pasar tiempo juntos & .589 & .876 \\
\hline 21 & Mi familia se distingue por sus relaciones armoniosas & .538 & .879 \\
\hline 3 & $\begin{array}{l}\text { En mi familia se buscan actividades recreativas en las que } \\
\text { todos podamos participar }\end{array}$ & .532 & .880 \\
\hline 30 & En mi familia se platican cosas entre hermanos & .464 & .884 \\
\hline \multicolumn{4}{|c|}{ Conflicto (9 ítems) $\alpha=.848$} \\
\hline 4 & En mi familia los límites y reglas son poco claros & .618 & .827 \\
\hline 37 & En mi familia se dice una cosa y se hace otra & .591 & .830 \\
\hline 11 & $\begin{array}{l}\text { Los miembros de mi familia, manifestamos entre nosotros } \\
\text { sentimientos diferentes a los que verdaderamente sentimos }\end{array}$ & .633 & .826 \\
\hline 17 & En mi familia cada quien resuelve sus problemas como puede & .556 & .833 \\
\hline 24 & $\begin{array}{l}\text { Las reuniones de mi familia ponen en evidencia las adicciones } \\
\qquad \text { de algunos de sus miembros }\end{array}$ & .537 & .835 \\
\hline 10 & En mi familia se desconoce lo que cada uno espera del otro & .574 & .832 \\
\hline 26 & $\begin{array}{l}\text { Mi familia emplea el doble mensaje, se pide una cosa cuando } \\
\text { en realidad se quiere se haga otra }\end{array}$ & .555 & .834 \\
\hline 5 & $\begin{array}{l}\text { Los integrantes de mi familia, mostramos desinterés por los } \\
\text { demás }\end{array}$ & .533 & .836 \\
\hline 2 & $\begin{array}{l}\text { En mi familia nadie sigue la disciplina establecida por los } \\
\text { papás }\end{array}$ & .495 & .840 \\
\hline \multicolumn{4}{|c|}{ Diversión (5 ítems) $\alpha=.791$} \\
\hline 20 & $\begin{array}{l}\text { En mi familia cualquier evento es bueno para celebrar e invitar } \\
\text { a nuestros amigos }\end{array}$ & .645 & .726 \\
\hline 22 & En mi familia nos agrada reunirnos con nuestros amigos & .638 & .728 \\
\hline 13 & $\begin{array}{l}\text { En mi familia, nos gusta organizar convivios y fiestas } \\
\text { familiares }\end{array}$ & .547 & .758 \\
\hline 18 & Mi familia tiene muchos amigos & .538 & .761 \\
\hline 28 & $\begin{array}{l}\text { Mi familia asiste a reuniones sociales (fiestas, bodas, bautizos, } \\
\text { cumpleaños) }\end{array}$ & .480 & .778 \\
\hline \multicolumn{4}{|c|}{ Hostilidad (4 ítems) $\alpha=.721$} \\
\hline 35 & $\begin{array}{l}\text { En mi familia, acostumbramos criticar a la persona que se } \\
\text { encuentra ausente }\end{array}$ & .537 & .645 \\
\hline 33 & $\begin{array}{l}\text { En mi casa los miembros de la familia ocultamos lo que nos } \\
\text { desagrada de los demás }\end{array}$ & .545 & .638 \\
\hline 7 & Los miembros de mi familia nos criticamos unos a otros & .523 & .651 \\
\hline 32 & $\begin{array}{l}\text { A los miembros de mi familia nos desagrada convivir con } \\
\text { personas ajenas a nuestra familia }\end{array}$ & .438 & .701 \\
\hline \multicolumn{4}{|c|}{ Coaliciones (3 ítems) $\alpha=.660$} \\
\hline 12 & $\begin{array}{l}\text { En mi familia los hijos se ponen de acuerdo entre ellos, para } \\
\text { obtener alguna ganancia de mi pareja y de mí }\end{array}$ & .502 & .523 \\
\hline 25 & $\begin{array}{l}\text { Mis hijos y mi pareja se unen para obtener algún beneficio de } \\
\text { mi persona }\end{array}$ & .465 & .571 \\
\hline 39 & $\begin{array}{l}\text { Mis hijos y yo nos ponemos de acuerdo para obtener algún } \\
\text { beneficio de mi pareja }\end{array}$ & .446 & .597 \\
\hline
\end{tabular}

CETC: Correlación elemento total corregido; $a$ :[e]: Alfa de Cronbach si se

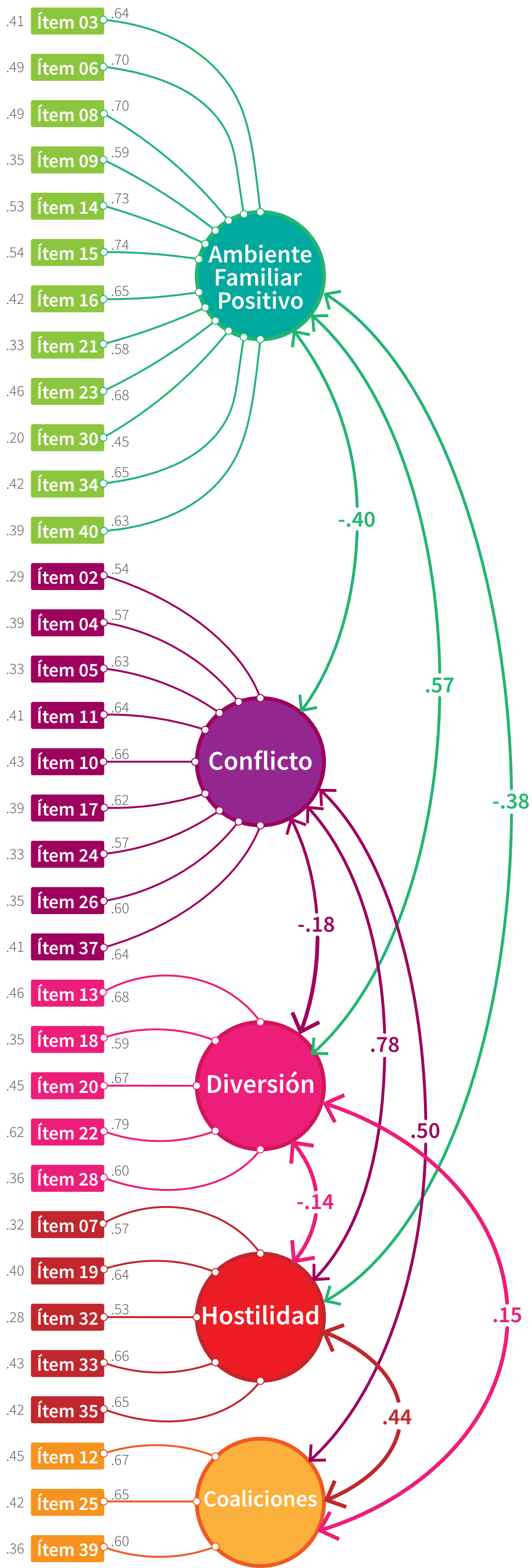

Gráfico 1 Modelo Estructural Confirmatorio de la escala de

funcionamiento familiar 


\section{Escala de funcionamiento familiar}

Propiedades psicométricas modificadas en una muestra mexicana

\section{Discusión}

La familia, como unidad y sistema, se desplaza en un continuo que incluye patrones de interacción que facilitan o entorpecen el movimiento y el cambio, lo cual se refleja en los factores y en los ítems que integran la escala. El objetivo de esta investigación fue obtener las propiedades psicométricas de la EFFAMO, en tanto se modificaron ítems y se incluyeron otros relacionados con dimensiones teóricas del funcionamiento familiar que no fueron contempladas en la primera versión (García Méndez, Rivera Aragón, Reyes Lagunes, \& Díaz Loving, 2006). Para ello se realizó un análisis factorial exploratorio con el que se obtuvo una escala conformada por cinco factores: ambiente familiar positivo, conflicto, diversión, hostilidad y coaliciones. Esta versión difiere de la primera (que se compone de cuatro factores y 45 ítems). Los análisis de consistencia interna realizados mediante el Alpha de Cronbach indicaron ser adecuados, debido a que estimaron por encima de $\mathbf{0 . 6 0}$, (Streiner, 2003), sugiere que las puntuaciones se mantengan por arriba del valor mencionado.

Una vez realizados los análisis psicométricos, se corroboró la estructura factorial de la escala mediante el análisis factorial confirmatorio. Con este análisis se obtuvieron los índices de ajuste adecuados. Estos resultados permitieron explicar la correlación entre las variables latentes y la asociación de éstas y sus correspondientes variables observadas, lo que es un indicador de que la escala puede ser utilizada para medir el funcionamiento familiar.

Los ítems de la EFFAMO tuvieron estimaciones paramétricas iguales o mayores a 0.402, (Nunnally, 1967/2013) sugiere que para la selección de los ítems el punto de corte sea de $\mathbf{0 . 2 5}$, por lo que los valores obtenidos fueron adecuados. El primer factor, Ambiente Familiar Positivo se mantiene. Los 11 ítems que lo integran se relacionan con el respeto, la expresión de afecto, la cercanía, la colaboración, la armonía y la comunicación entre los integrantes de la familia. (Olson, 1991) establece que estas habilidades positivas facilitan poder compartir las necesidades y preferencias de la familia, así como la generación de alternativas de solución de problemas cuando éstos se presentan.

El segundo factor denominado 'conflicto' forma parte del factor dos de la primera versión de la escala -hostilidad/evitación del conflicto- que se dividió en dos: Conflicto (factor dos) y Hostilidad (factor cuatro). Conflicto se integra por nueve ítems que hacen referencia a la desvinculación, la confusión, la falta de cooperación y comunicación, el desinterés y la desorganización en la familia. Así mismo, se relaciona con las habilidades negativas que reducen la capacidad de los miembros de la familia para compartir sus sentimientos, elemento que dificulta el movimiento cuando es requerido. (Olson, 2000)

El factor tres, Diversión, es nuevo. Éste se compone de cinco ítems referentes a la convivencia mediante reuniones sociales con la familia y los amigos. Es importante mencionar que Zabrieskie (2001), reporta que la recreación familiar se relaciona con la satisfacción y la cohesión familiar. Estefactor se relacionaal hecho quelas celebraciones familiares son un elemento que contribuye a la estabilización de la familia y en el que se avala su sistema de creencias compartidas. (Bennett, 1991)

El factor cuatro, Hostilidad, contiene cuatro ítems relacionados con la crítica, la incomodidad y la indiferencia, lo que dificulta el acercamiento entre los integrantes de la familia. Se reporta que las relaciones hostiles entre los padres contribuyen en la presencia de problemas en los hijos y en los diferentes niveles del sistema familiar. (Fainsilber Katz \& Woodin, 2002)
El factor cinco, Coaliciones, -al igual que diversión- es un factor nuevo, integrado por tres ítems que hacen énfasis en la alianza de dos o más integrantes de la familia, lo que puede dañar el funcionamiento familiar. La alianza de uno de los padres con un hijo genera que se aleje de su pareja y haya mayor acercamiento hacia éste, a la vez que el otro padre forma una relación más distante con dicho hijo. (Klever, 2009)

Estos resultados coinciden con la teoría que plantea que el funcionamiento familiar sucede en un continuo (Olson, 2000; Russel, 1979; Schrodt, 2005), en el que intervienen aspectos positivos y negativos. Entre los positivos se encuentran las habilidades de comunicación y solución de problemas, especialmente cuando se considera que la relación puede ser conflictiva entre los miembros de la familia (Martin \& Martin, 2000). Por otro lado, las habilidades negativas de comunicación como el mensaje ambiguo y la crítica, reducen al mínimo la capacidad de los miembros de la familia para compartir sus sentimientos (Olson, 1991), reducen la posibilidad de apoyo y genera una pobre mediación para resolver los problemas. (Beavers \& Voeller, 1983)

Resultado de este estudio se obtuvo un instrumento válido y confiable para evaluar el funcionamiento familiar, el cual puede ser enriquecido con futuras investigaciones en diferentes contextos y empleando otros instrumentos similares.

\section{Conflicto de intereses}

Los autores declaran no tener conflicto de intereses.

\section{Referencias}

Ackerman, N. W. (1995). Diagnóstico y tratamiento de las relaciones familiares: psicodinamismos de la vida familiar [Teoría y métodos de la Psicología: Psicología de hoy] (10 ed., Vol. 4). Argentina: Hormé.

Anderson, S. A., \& Sabatelli, R. M. (2003). Family interaction: a multigenerational developmental perspective (3 ed.). Boston: Allyn \& Bacon. Obtenido de: http://trove.nla.gov.au/ork/20990964?\&version/d=40876594

Andolfi, M., Angelo, C., Menghi, P., \& Nicolò Corigliano, A. M. (2007). Detrás de la máscara familiar (3 ed.). Buenos Aires: Amorrortu.

Atri, Y., \& Zetune, R. (1987). Confiabilidad y validez del cuestionario de evaluación del funcionamiento familiar. Mexico: Universidad de las Américas.

Beavers, W. R., \& Voeller, M. N. (1983). Family models: Comparing the Olson circumplex model with the Beavers systems model. Family Process, 22(1), 85-97. DOI: 10.1111/j.1545-5300.1983.00085.x

Bennett, L. A. (1991). Identidad de la familia, ritual y mito: una perspectiva cultural de las transiciones en el ciclo vital.

David Barrett, T., Kertesz, J., Rotkirch, A., Ghosh, A., Bhattacharya, K., Monsivais, D., \& Kaski, K. (2016). Communication with Family and Friends across the Life Course. (R. Lambiotte, Ed.) PLoS ONE, e0165687. DOI:10.1371/journal.pone.0165687

de la Fuente Fernández, S. (2011). Análisis Factorial. Guía de clase, Universidad Autónoma de Madrid, Fac. Ciencias Económicas y Empresariales, Madrid. Obtenido de: http://wWw.fuenterrebollo.com/Economicas/ECONOMETRIA/ MULTIVARIANTE/FACTORIAL/analisis-factorial.pdf

Díaz Guerrero, R. (1994). Psicología del méxicano: Descubrimiento de la etnopsicología (6 (1999) ed.). México D.F.: Trillas S.A. de C.V. Obtenido de https://es.scribd.com/document/322927542/Psicologia-Del-MexicanoRogelio-Diaz-Guerrero

Díaz Guerrero, R. (2003). Bajo las garras de la cultura: Psicología del Mexicano (1 ed., Vol. 1). México D.F.: Trillas. 
Díaz Loving, R. (2004). Una aproximación bio-psico-socio-cultural a la procuración de conductas sanas y al alejamiento de conductas nocivas en la relación de pareja. Revista Mexicana de Psicología, 21(2), 157-165.

Donalek, J. G. (2009). The family research interview. Nurse Researcher, 16(3), 21-28. DOI: $10.7748 /$ nr2009.04.16.3.21.c6943

Epstein, N. B., Baldwin, L. M., \& Bishop, D. S. (1983). The McMaster Family Assessment Device. Journal of Marital and Family Therapy, 9(2), 171-180. DOI: 10.1111/j.1752-0606.1983.tb01497.x

Everri, M., Fruggeri, L., \& Molinari, L. (2014). Microtransitions and the Dynamics of Family Functioning. Integrative Psychological and Behavioral Science, 48(1), 61-78. DOI: 10.1007/s12124-013-9248-9

Fainsilber Katz, L., \& Woodin, E. M. (2002). Hostility, Hostile Detachment, and Conflict Engagement in Marriages: Effects on Child and Family Functioning. Child development, 73(2), 636-652. DOI: 10.1111/1467-8624.00428

García Cortés, J. M., García Méndez, M., \& Rivera Aragón, S. (2015). Potencial resiliente en familias con adolescentes que consumen y no consumen alcohol. Acta Colombiana de Psicología, 18(2), 163-172. DOI:10.14718/ACP.2015.18.2.14

García Méndez, M., Rivera Aragón, S., Reyes Lagunes, I., \& Díaz Loving, R. (2006). Construcción de una escala de funcionamiento familiar. Revista Iberoamericana de Diagnóstico y Evaluación Psicológica, 2(22), 91-110. Obtenido de: $h t t p: / / w w w$.aidep.org/sites/default/files/articles/R22/R225.pdf

Garst, B. A., Baughman, S., Franz, N. K., \& Seidel, R. W. (2013). Strengthening families: exploring the impacts of family camp experiences on family functioning and parenting. Journal of Experiential Education, 36(1), 65-77. DOI: $10.1177 / 1053825913481582$

Hoffman, L. (1992). Fundamentos de la terapia familia: Un marco conceptual para el cambio de sistemas (2 [es] ed.). México D.F.: Fondo de Cultura Económica de México S.A. de C.V. Obtenido de: http://www. verticespsicologos.com/sites/default/files/Fundamentos-de-la-terapiafamiliar.pdf

Hu, L.-t., \& Bentler, P. M. (1999). Cutoff criteria for fit indexes in covariance structure analysis: Conventional criteria versus new alternatives. Structural Equation Modeling: A Multidisciplinary Journal, 6(1), 1-55. DOI: https:/doi. org/10.1080/10705519909540118

Klever, P. (2009). The primary triangle and variation in nuclear family functioning. Contemporary Family Therapy, 31(2), 140-159. DOI:10.1007/s10591-0089082-2

Larraín, M. E., Zegers, B., Díez, I., Trapp, A., \& Polaino Lorente, A. (2003). Validez y confiabilidad de la versión española de la escala del estilo de funcionamiento familiar (EFF) de Dunst, Trivette \& Deal para el Diagóstico del Funcionamiento Familiar en la población chilena. Psykhe, 12(1), 195-211. Obtenido de: http://www.psykhe.cl/index.php/psykhe/article/ view/337/317

Larson, R. W., \& Almeida, D. M. (1999). Emotional Transmission in the Daily Lives of Families: A New Paradigm for Studying Family Process. Journal of Marriage and the Family, 61(1), 5-20. DOI: 10.2307/353879

Lo Presti, A., D’Aloisio, F., \& Pluviano, S. (2016). With a little help from my family: A mixed-method study on the outcomes of family support and workload. Europe's Journal of Psychology, 12(4), 584-603. DOI:10.5964/ejop.v12i4.1159

Martin, D., \& Martin, M. (2000). Understanding Dysfunctional and Functional Family Behaviors for the At-Risk Adolescent. Adolescence, 35(140), 35, 785-793. Obtenido de: https://www.questia.com/library/journal/1G1-70777839/ understanding-dysfunctional-and-functional-family

Matheson, K., Kelly, O., Cole, B., Tannenbaum, B., Dodd, C., \& Anisman, H. (2005). Parental bonding and depressive affect: The mediating role of coping resources. Social Psychology, 44(3), 371-395. DOI:10.1348/014466605×37477

McGoldrick, M., \& Gerson, R. (1993/2009). Genogramas en la evaluación familiar (2 ilustrada, reimpresa ed.). (C. Ferrari, Trad.) Gedisa.

Minuchin, S. (1974/1995). Familias y terapia familiar (8[es] 1[mx] ed., Vol. 2). (V. Finchman, Trad.) Boston: Gedisa Mexicana, S.A. [Harvard College]. Obtenido de: https://es.scribd.com/doc/64215656/Familia-y-TerapiaFamiliar-Minuchin

Moos, R. H., Insel, P. M., \& Humprey, B. (1974). Preliminary manual for family environment scale, work environment scale, group environment scale. Michigan University. Palo Alto: Consulting Psychologists Press.
Nunnally, J. C. (1967/2013). Teoria psicometrica (3 Rustica ed.). México D.F.: Trillas.

Olson, D. H. (1991). Tipos de familia, estrés familiar y satisfacción con la familia: una perspectiva del desarrollo familiar. En C. Falicov (Ed.), Transiciones de la familia. Continuidad y cambio en el ciclo vital (págs. 99-128). St. Paul: Amorrort [Buenos aires].

Olson, D. H. (2000). Circumplex Model of Marital and Family Sytems. Journal of Family Therapy, 22(2), 144-167. DOI:10.1111/1467-6427.00144

Olson, D. H. (2011). FACES IV and the circumplex model: Validation study. St. Paul. doi: doi: 10.1111/j.1752-0606.2009.00175.x

Olson, D. H., Russell, C. S., \& Sprenkle, D. H. (1983). Circumplex Model of Marital and Family Systems: VI. Theoretical Update. Family Process, 22(1), 69-83. DOI: https:/doi.org/10.1111/j.1545-5300.1983.00069.x

Palomar, J. (1999). Relación entre el funcionamiento familiar y la calidad de vida en familias con un miembro alcohólico. Salud Mental, 22(6), 13-21. Obtenido de http://www.revistasaludmental.mx/index.php/salud mental/article/ view/770; http://www.imprf-cd.gob.mx/pdf/sm2206/sm220613.pdf

Pernice Duca, F. (2010). Family Network Support and Mental Health Recovery. Journal of Marital and Family Therapy, 36(1), 13-27. DOI:10.1111/j.1752-0606.2009.00182.x

Rea Amaya, A. C., Acle Tomasini, G., Ampudia Rueda, A., \& García Mendez, M. (2014). Caracterización de los conocimientos de las madres sobre la discapacidad de sus hijos y su vínculo con la dinámica familiar. Acta Colombiana de Psicología, 11(3), 91-103. DOI: 10.14718/ACP.2014.17.1.10

Rivera Heredia, M. E., \& Andrade Palos, P. (2010). Escala de evaluación de las Relaciones Intrafamiliares (E.R.I.). Uaricha Revista de Psicología, 7(14), 12-29. Obtenido de https://www.academia.edu/6596473/Escala de evaluaci\%C3\%B3n de las Relaciones Intrafamiliares

Rojas Ramírez, A. T., \& García Méndez, M. (2016). Funcionamiento familiar como predictor de la alexitimia de acuerdo al índice de masa corporal. Acta de Investigación Psicológica, 6(2), 6(2), 2469-2476. DOI:10.1016/j.aipprr.2016.06.010

Roque Hernández, M. d., \& Acle Tomasini, G. (2013). Resiliencia materna, funcionamiento familiar y discapacidad intelectual de los hijos en un contexto marginado. Universitas Psychologica, 12(3), 811-820. DOI: 10.11144/Javeriana.UPSY12-3.rmff

Russel, C. S. (1979). Circumplex Model of Marital and Family Systems: III. Empirical Evaluation With Families. Family Process, 18(1), 29-45. DOI:10.1111/i.1545-5300.1979.00029.x

Schrodt, P. (2005). Family Communication Schemata and the Circumplex Model of Family Functioning. Western Journal of Communication, 69(4), 359-376. DOI:10.1080/10570310500305539

Streiner, D. L. (2003). Being Inconsistent About Consistency: When Coefficient Alpha Does and Doesn't Matter. Journal of Personality Assessment, 80(3), 217222. DOI:10.1207/S15327752JPA8003 01

Toledano Toledano, F. (2010). Migración y funcionamiento familiar: su relación con las normas socioculturales. Universidad Nacional Autónoma de México, Maestría en Trabajo Social. México D.F.: Universidad Nacional Autónoma de México.

Vandeleur, C. L., Jeanpretre, N., Perrez, M., \& Schoebi, D. (2009). Cohesion, Satisfaction With Family Bonds, and Emotional Well-Being in Families With Adolescents. (V. McBride Murry, Ed.) Journal of Marriage and Family, 71(5), 1205-1219. DOI:10.1111/j.1741-3737.2009.00664.x

Weston, R., \& Qu, L. (2014). Trends in family transitions, forms and functioning: Essential issues for policy development and legislation. Family Matters, 95, 79-84. Obtenido de: https://aifs.gov.au/publications/family-matters/ issue-95/trends-family-transitions-forms-and-functioning

Williams, K. (2003). Has the Future of Marriage Arrived? A Contemporary examination of gender, marriage, and psychological well-being. Journal of Health and Social Behavior, 44(4), 470-487. Obtenido de http://www. jstor.org/stable/1519794; https://www.ncbi.n/m.nih.gov/pmc/articles/ PMC4018193/

Zabrieskie, R. B. (2001). The Influences of Family Leisure Patterns on Perceptions of Family Functioning. Family Relations, 50(3), 281-289. DOI: https:/doi. org/10.1111/j.1741-3729.2001.00281.x

Zafra, J. (2016). The Use of Structural Family Therapy With a Latino Family: A Case Study. Journal of Systemic Therapies, 35(4), 11-21. DOI:10.1521/jsyt.2016.35.4.11 